\title{
Minds and machines: Computers and cognition in a liberal arts context
}

\author{
STEVEN CUSHING \\ Stonehill College, North Easton, Massachusetts
}

\begin{abstract}
A series of simple experiments is used to demonstrate to undergraduates that mental phenomena are real, that they can be studied experimentally, and that they can be modeled insightfully in computational (i.e., information-processing) terms. Each experiment illustrates a basic fact, concept, or principle of cognitive science: the formal character of algorithms; creativity and the variants of the Turing test; limitations on human memories; the use of cognitive strategies; heuristic techniques of artificial intelligence; formal grammars and their associated parsers; societal and anthropological dimensions of mind; and degrees of logicality in human reasoning. Students are also taught the essentials of PROLOG, a programming language that is based explicitly on formal logic, incorporating such notions as fact, database, and query, thereby lending itself readily to the description of complex relational networks of a sort not commonly expected to be amenable to computer analysis.
\end{abstract}

This paper reviews a one-semester course, Minds and Machines, designed and taught for the past two years in order to demonstrate to undergraduate students in nontechnical majors how traditional philosophical problems concerning knowledge, cognition, language, and human nature can be fruitfully investigated with computer-related concepts and techniques. Emphasis is placed on three key notions: that mental phenomena are real, that they can be studied experimentally, and that they can be modeled insightfully in computational (i.e, information-processing) terms.

The first 9 weeks of the course revolve around a series of experiments that are conducted in class first, and then as homework assignments by the students on their own. Though simple in concept, each experiment illustrates a basic fact or principle drawn from one of the disciplines that are generally recognized as having contributed substantially to the development of cognitive science: computer science, philosophy, psychology, artificial intelligence, linguistics, and anthropology. Background readings are drawn from relevant books (e.g., Gardner, 1985; Weizenbaum, 1976), journals (e.g., Science), and current magazines (e.g., Science News and Scientific American) that are not excessively technical.

The last 5 weeks of the course are used to introduce the students to PROLOG, a programming language that is based explicitly on formal logic, in order to give them a feel for the kind of computer programming that goes into modeling of mental phenomena. PROLOG incorporates such notions as fact, database, query, and the like

I wish to thank Muriel Vasconcellos and Josette M. Coughlin for comments on my presentation that helped in its preparation for publication. Correspondence may be addressed to Steven Cushing, Department of Mathematics and Computer Science, Stonehill College, North Easton, MA 02357. and readily lends itself to the description of complex relational networks (e.g., the family relationships of the Greek gods) that are common in literary and philosophical discourse, but that are not ordinarily expected by humanities majors to be amenable to computer analysis.

\section{THE EXPERIMENTS}

In what follows, I describe and explain the rationale for the cognitive experiments that form the core of the course and that prepare the students for the programming unit.

\section{Experiment 1: "Mind-Reading"}

A mind-reading experiment is done, in which the instructor guesses, for each of the students, a number that the student has chosen. After being told to choose a number from 1 to 10 , the students are instructed to perform various arithmetic operations, after which they are asked what they have obtained. They are then told, always correctly, what numbers they originally chose. The students generally do not believe that the instructor has actually read their minds (nor do most of their friends, when they do the experiment as a homework assignment), but they are always at a loss to explain how one might have determined their numbers, their best guess usually being "some kind of mathematical trick." The trick, of course, is simple algebra, in which one applies to the variable $x$ the same operations that one instructs the students to apply to their chosen number. The final expression, when combined with each of their results, becomes a simple equation that is easily solved mentally for $\boldsymbol{x}$ (see Figure 1).

Despite its simplicity, this experiment opens the door to a discussion of the most fundamental concept of computer science, namely, the formal character of algorithms (i.e., the fact that an algorithm can be applied to all enti- 
Instructions to Students

1. Choose a number.

2. Add 1.

3. Multiply the result by itself.

4. Subtract the square of the original number.

5. Tell me your current result.

6. Your original number is 6 .
Sample Student's Results

6

7

49

$49-36=13$

13
Mental Calculation

$\mathbf{x}$

$x+1$

$(x+1)^{2}=x^{2}+2 x+1$

$2 x+1$

$2 x+1=13$

$x=6$

Figure 1. Sample calculation for Experiment 1.

ties of the appropriate sort [in this case, numbers] without regard to the specific reference of the symbols in terms of which the algorithm is expressed). Arithmetic expresses the particular properties of individual numbers (e.g., only the number 6 has the property of being the product of 2 and 3); algebra, in contrast, expresses the universal properties of numbers in general [e.g., every number $x$ has the property that $\left.(x+1)^{2}=x^{2}+2 x+1\right]$. It is this property of formality that makes algorithms implementable as computer programs and that enables machines to produce humanly significant results by merely manipulating symbols in accordance with fixed rules.

\section{Experiment 2: "Story-Guessing"}

Six student volunteers are solicited to leave the room, after being told that those who remain will make up a story for them to guess by asking only yes/no questions. After the guessers leave, the remaining students are told that there will really be no story at all. If the guessers ask a question that ends in a vowel $(a, e, i, o, u, y)$ the answer will be yes; if the question ends in a consonant, the answer will be no. (It is important that $y$ be considered a vowel in this experiment, because someone invariably asks the question, "Is there really a story?" If $y$ were considered a consonant, this would immediately end the experiment.) The guessers end up constructing a story of their own, based on their own interests and backgrounds, in response to the (in effect) random answers given to their questions, without even realizing that they are doing so. Discussion centers on the differences between the roles of guesser and answerer and the likelihood that a computer could successfully play one of these roles.

The experiment thus provides the basis for a discussion of creativity, as it relates to the distinction between human beings and machines in general, and to the variant of the Turing test in particular (i.e., Turing's suggestion that a computer must be considered to have human intelligence if a human observer cannot distinguish its answers to yes/no questions from those that would be given by a human answerer; Turing, 1963). Zadeh (1986) has suggested replacing the requirement of yes/no answers with that of "summarization in your own words," thereby placing creativity, rather than binary choice, at the center of the distinction between human beings and machines.

\section{Experiment 3: "Memory Limits"}

Two student volunteers are solicited to investigate limitations on human memories. One student is given a random-number table and asked to read out random-length sequences of digits for the other student to repeat, with careful track being kept of the lists read back in response and, in particular, of the kinds of errors made. The roles are then reversed and the experiment repeated.

This provides the basis for a discussion of Miller's magic number $7 \pm 2$, which reflects human short-term memory limits (Miller, 1956), of cognitive limits in general and how they might arise from the basic architecture of the mind and brain as computational devices, and of strategies that are used to overcome them, such as chunking (e.g., remembering a 10-digit telephone number as a 3-element sequence of 3 digits, 3 digits, and 4 digits) and linguistic recoding (e.g., remembering the 16digit sequence 1861186519141918 as a 6-item sequence-actually, 3 items, consisting of 2 items, 3 items, and 1 item, respectively-“"Civil-War World-WarOne Dates"'). Appropriate analogies can then be drawn to the notion of data structures in computer programming, a device that accomplishes essentially the same result.

\section{Experiment 4: "Pattern-Guessing"}

One student volunteer is solicited to further investigate the use of cognitive strategies by participating in one or more games of Mastermind (Flood, 1988; Hentenryck, 1988; Yue, 1986). In this game, one player chooses a pattern of shapes and/or colors, which the other player must then figure out by guessing at what the target pattern might be and receiving answers as to how many, but not which, shapes or colors are correct (see Figure 2).

First the student tries to guess the target pattern; then the student is asked to explain his or her strategy, if any; this strategy is then analyzed for effectiveness. Next the student chooses the target pattern, and the standard strate- 


Response
to Guesser
$\begin{aligned} & \text { 1. One pair correctly matched } \\ & \text { and in the correct position. } \\ & \text { One pair correctly matched } \\ & \text { but in an incorrect position. } \\ & \begin{array}{l}\text { One item correct in the correct position } \\ \text { but incorrectly matched. }\end{array} \\ & \begin{array}{l}\text { 1. Guessed pair in Position } 3 \\ \text { from left is correct. }\end{array} \\ & \text { 2. Guessed pair in Position } 1 \text { from left } \\ & \text { should be in Position } 2 \text { from left. }\end{aligned}$
3. Guessed item in lower position 2
from left is correct.

Figure 2. Sample Mastermind move for Experiment 4.

gies of successive scanning (assuming a pattern as a hypothesis and then comparing it to possible counterexamples), conservative focusing (assuming a pattern as a hypothesis and then varying it one attribute at a time), and focus gambling (assuming a pattern as a hypothesis and then varying it more than one attribute at a time) are deliberately used and explained (Bruner, Goodnow, \& Austin, 1956). This provides the basis for a more general discussion of cognitive strategies, cognitive maps (Tolman, 1932), and the like, thereby laying the foundation for the introduction of the notion of heuristics (i.e., the formalized "rules of thumb" that are used as the basis for game-playing and more general decision-making programs in artificial-intelligence systems).

\section{Experiment 5: "Machine Emulation"}

Decision trees, search strategies, evaluation functions, and other techniques of artificial intelligence (AI) are explained as specific heuristics, by applying the two-ply minimax search strategy to the game of tic tac toe (Bramer, 1983; Levy, 1983). In accordance with this strategy, a tree of all possible moves and immediate countermoves is constructed, and the resulting game configurations are evaluated at each turn in the game, in order to determine how near to a victory each configuration is and thus to determine the best next move (see Figure 3 ).

The contrast between the rapidity and apparent nearspontaneity of actual human game-playing and the much slower-in fact, boring - pace that results from deliberate use of a conscious computational strategy-indeed, a strategy that appears to underlie the extreme rapidity and near-spontaneity that is exhibited by game-playing computers-provides the framework for a discussion of conscious versus nonconscious knowledge and planning in the general distinction between human beings and machines.

\section{Experiment 6: "Tag Questions"}

The notion of a tag question is explained, and the students are then asked to respond with appropriate tags to examples such as those given in Figure 4 (Langendoen, 1970). They soon see that nothing they have learned about English is of any help here, because the examples get so bizarre that even their own linguistic intuitions are quickly strained.

Students find the results of this experiment particularly intriguing, in view of the emphasis that has always been placed in their previous education on the notion of "correct" grammar. It provides the basis for a discussion of descriptive as opposed to prescriptive grammar and of what it takes to describe grammar formally, including finite-state, phrase-structure, and transformational grammars, and their associated parsers, the formal devices that are used as the basis of many natural-language processing and machine-translation systems (Winograd, 1983).

\section{Experiment 7: The "Rumor Mill"}

Students are arranged in a random numerical order. A story is told to Student 1 , who repeats it to Student 2, who repeats it to Student 3, and so on. Each student is also asked to write the story down, after repeating it. The last student recites the story aloud, after which it is compared to the original version, and each student is asked to comment on how closely this version compares to the one that he or she wrote down. Invariably, the story has undergone substantial transmutation during its journey among 


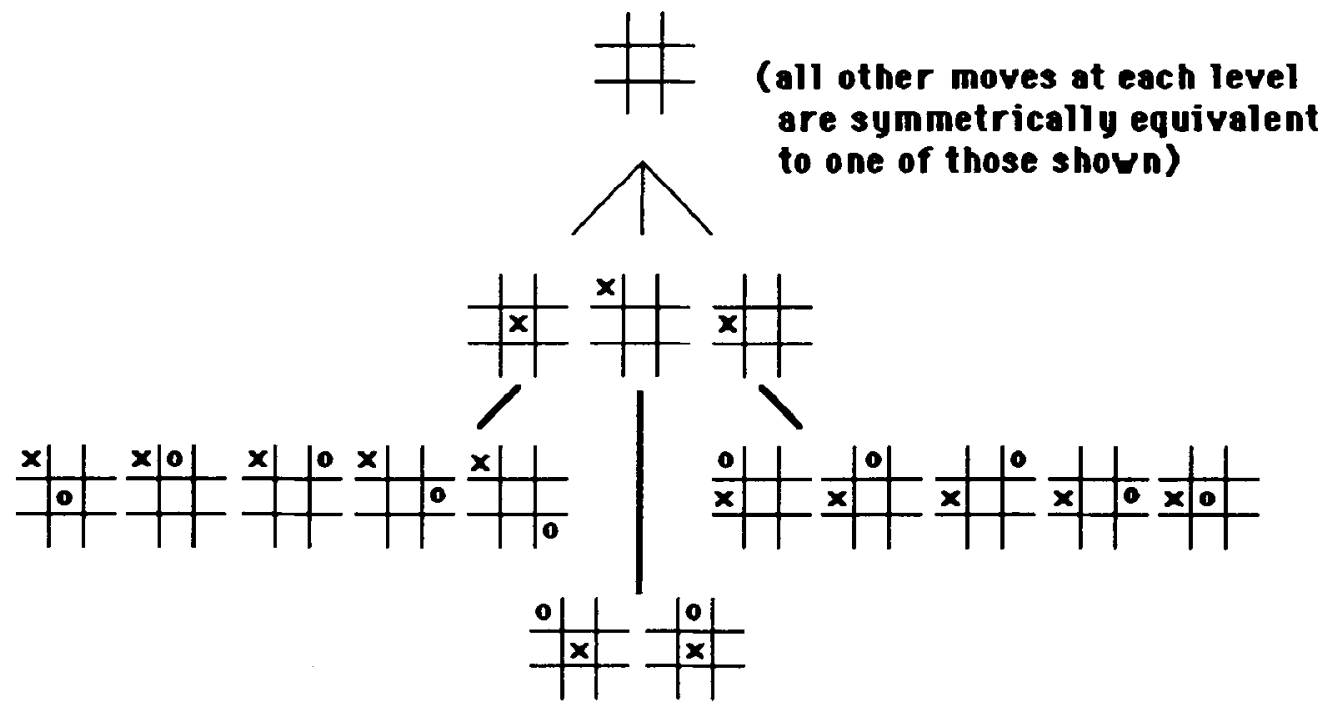

Figure 3. Two-ply tree of possible moves for determining the best initial move in Experiment 5.

the students, with sometimes no substantive resemblance whatsoever between the initial and final versions.

This provides the basis for a discussion of the societal and anthropological dimensions of mind (Cushing, in press $\mathrm{a}$, in press $\mathrm{c}$ ), particularly as regards culture formation, mythmaking, and the spread of rumor and gossip. Such aspects of miscommunication in human-human interaction (Cushing, in press b) can then be extended into a discussion of the problems of human-machine interaction, machine-machine networking, and the importance of adequate documentation in designing and implementing an actual computer system.

\section{Experiment 8: "Mental Models"}

Students are told stories of varying degrees of complexity and then asked questions as to the existence of mentioned or unmentioned entities, along the lines of Cushing $(1982,1984)$, and the validity of various kinds of inferences, along the lines of Johnson-Laird (1983).

The former provides the basis for a discussion of presupposition and default (i.e., the tendency of human minds to take things for granted in appropriate contexts; see Cushing, 1987, in press d), and of the role these play in inferencing programs such as those used in expert systems. The latter provides the basis for a discussion of the
1. S: I like ice cream,

T: don't I?

2. S: You don't seem to understand me,

T: do you?

3. S: Your father can do a hundred push-ups,

T: can't he?

4. S: Louise is intelligent,

$\mathrm{T}$ : isn't she?

5. S: We won't tolerate such nonsense,

T: will we?

6. S: The sky looks threatening,

T: doesn't it?
1. S: I have to go home now,

$T:$

????

2. S: There's no chalk on the rack, T: ????

3. S: I may not see you tomorrow, T: ????

4. S: I'm going to the store now, $T$ : ????

5. S: No one watches TV any more, $\mathrm{T}:$ ????

6. S: Either John or Sue will stay, T: ???? 
degree of logicality of human reasoning and the role played in it by semantic models (i.e., formal representations of the entities and situations about which people reason). Also provided is an opening for some discussion of fuzzy logic (i.e, a formalized logic that recognizes degrees of truth and logical validity; see Schmucker, 1984) and the extent to which this might be needed in the modeling of cognitive processes.

\section{EVALUATION}

There are several factors that could be taken as criteria for evaluating the success of this course: class attendance, level of class discussion, student enthusiasm for participation in class experiments, and quality of assignments, papers, and exams. Class attendance tends to be high for a course of this sort, or, indeed, for any course at all on days when experiments are to be performed. The level of class discussion tends to be lower than one would like-a result, it would seem, more of the students' insufficient backgrounds than of their lack of interest in the subject matter. Enthusiasm is also low at first with respect to volunteering for in-class experiments, but it tends to increase significantly once the experiments get going, and to persist through the assignments as well. The actual experience of seeing minds in motion apparently overcomes students' general reluctance to take chances that might jeopardize grades. In the interest of maximizing their motivation, the students are given substantial leeway in choice of term-paper topics, which range from the history of the microprocessor and its influence on AI to subliminal seduction in media advertising. Any topic relating to the mind is permitted, as long as significant connections are made in the papers to the material discussed in class. The students themselves appear to be satisfied with the course, stressing, when asked, its participatory character and its emphasis on creative thinking, rather than rote memorization.

\section{REFERENCES}

Bramer, M. A. (1983). Computer game-playing: Theory and practice. Chichester, England: Ellis Horwood.

Bruner, J. S., GoOdNow, J., \& AUSTIN, G. (1956). A study of thinking. New York: Wiley.
CUSHING, S. (1982). Quantifier meanings: A study in the dimensions of semantic competence. Amsterdam: North-Holland.

Cushing, S. (1984). Dynamic model selection in the interpretation of discourse. In L. Vaina \& J. Hintikka (Eds.), Cognitive constraints on communication: Representations and processes (pp. 351-361). Dordrecht, The Netherlands: Reidel.

Cushing, S. (1987). Two explanatory principles in semantics. In L. Vaina (Ed.). Matters of intelligence (pp. 241-253). Dordrecht, The Netherlands: Reidel.

Cushing, S.(in press a). Explaining a missing modal meaning: Ideology and paradigm as pragmatic parameter. In J. Verschueren (Ed.), Levels of linguistic adaptation: Selected papers from the 1987 International Pragmatics Conference. Philadelphia and Amsterdam: Benjamins.

CusHing, S. (in press b). Language and communication-related problems of aviation safety. Washington, DC: U.S. Department of Education.

Cushing, S. (in press c). Prototypical considerations on modal meanings. In S. L. Tsohatzidis (Ed.), Meanings and prototypes: Studies on linguistic categorization. London and New York: Routledge \& Kegan Paul.

Cushing, S. (in press d). Some tri-valent quantifiers from natural language: Explanatory and methodological principles of semantic inquiry. Communication \& Cognition.

FLoOD, M. M. (1988). Sequential search strategies with mastermind variants-Part 1. Joumal of Recreational Mathematics, 20, 105-126.

GARDNER, H. (1985). The mind's new science: A history of the cognitive revolution. New York: Basic Books.

Hentenryck, P. V. (1988, January). A constraint approach to mastermind in logic programming. SIGART Newsletter, pp. 31-35.

Johnson-LAIRD, P. N. (1983). Mental models: Towards a cognitive science of language, inference, and consciousness. Cambridge, MA: Harvard University Press.

LANGENDOEN, D. T. (1970). Essentials of English grammar. New York: Holt, Rinehart \& Winston.

Levy, D. (1983). Computer gamesmanship. New York: Simon \& Schuster.

MilLeR, G. A. (1956). The magical number seven, plus or minus two: Some limits on our capacity for processing information. Psychological Review, 63, 81-97.

SCHMUCKer, K. J. (1984). Fuzzy sets, natural language computations, and risk analysis. Rockville, MD: Computer Science Press.

Tolman, E. C. (1932). Purposive behavior in animals and men. New York: Century.

TURING, A. M. (1963). Computing machinery and intelligence. In E. Feigenbaum \& J. Feldman (Eds.), Computers and thought. New York: McGraw-Hill. (Original work published 1950)

Weizendaum, J. (1976). Computer power and human reason. San Francisco: W. H. Freeman.

WINOGRAD, T. (1983). Language as a cognitive process: Vol. I. Syntax. Reading, MA: Addison-Wesley.

YUE, K.-B. (1986, October). Some heuristics for playing mastermind. SIGART Newsletter, pp. 21-22.

ZADEH, L. (1986, Spring). A computational approach to commonsense reasoning. Lecture at Boston University. 